\title{
Aqueous Cytokine Levels Are Associated With Progression of Peripheral Anterior Synechiae After Descemet Stripping Automated Endothelial Keratoplasty
}

\author{
Yuki Kusano ${ }^{1,2}$, Takefumi Yamaguchi ${ }^{1}$, Sota Nishisako ${ }^{1,3}$, Takehiro Matsumura ${ }^{1}$, \\ Masaki Fukui ${ }^{1}$, Kazunari Higa ${ }^{1,3}$, Toshihiro Inoue ${ }^{2}$, and Jun Shimazaki ${ }^{1,3}$ \\ ${ }^{1}$ Department of Ophthalmology, Tokyo Dental College, Ichikawa General Hospital, Chiba, Japan \\ ${ }^{2}$ Department of Ophthalmology, Faculty of Life Sciences, Kumamoto University, Kumamoto, Japan \\ ${ }^{3}$ Cornea Center Eye Bank, Tokyo Dental College, Ichikawa General Hospital, Chiba, Japan
}

Correspondence: Takefumi Yamaguchi, Department of Ophthalmology, Tokyo Dental College, Ichikawa General Hospital, 5-11-13, Sugano, Ichikawa-shi, Chiba 272-8513, Japan.

e-mail: yamaguchit@tdc.ac.jp

Received: June 6, 2021

Accepted: August 16, 2021

Published: September 9, 2021

Keywords: aqueous humor; glaucoma; cytokine; peripheral anterior synechiae; Descemet's stripping automated endothelial keratoplasty

Citation: Kusano Y, Yamaguchi T, Nishisako S, Matsumura T, Fukui M, Higa K, Inoue T, Shimazaki J. Aqueous cytokine levels are associated with progression of peripheral anterior synechiae after Descemet stripping automated endothelial keratoplasty. Transl Vis Sci Technol. 2021;10(11):12, https://doi.org/10.1167/tvst.10.11.12
Purpose: To evaluate the association between total protein and cytokine levels in aqueous humor $(\mathrm{AqH})$ and the progression of peripheral anterior synechiae (PAS) after Descemet stripping automated endothelial keratoplasty (DSAEK).

Methods: This prospective study included 146 consecutive patients who underwent DSAEK. Preoperative levels of total protein and 20 cytokines in $\mathrm{AqH}$ were measured. Using three-dimensional anterior segment optical coherence tomography, we assessed the iridotrabecular contact (ITC) area before and at 3, 6, and 12 months after DSAEK. Correlations between the total protein and cytokine levels in the AqH and ITC area were analyzed.

Results: ITC was observed in 47 eyes (32.2\%) after DSAEK. The ITC area increased from $2.00 \pm 4.42 \mathrm{~mm}^{2}$ preoperatively to $3.00 \pm 6.85 \mathrm{~mm}^{2}$ at 12 months. The total protein level in AqH was significantly higher in eyes with ITC progression than in those without $(1.45 \pm 1.03 \mathrm{mg} / \mathrm{mL}$ vs. $1.00 \pm 0.57 ; P=0.04)$ and was significantly positively correlated with the progression of ITC area after DSAEK (at 6 months, $r=0.311$ and $P=0.005$; at 12 months, $r=0.342$ and $P=0.0004)$. The ITC area at 12 months was significantly correlated with the preoperative AqH levels of interleukin-8 $(r=0.252 ; P=0.021)$, interferon$\gamma(r=0.318 ; P=0.009)$, and soluble intercellular adhesion molecule-1 $(r=0.292$; $P=0.004)$. Multivariate analyses showed that the total protein levels in $\mathrm{AqH}$ and the presence of preoperative ITC were significant risk factors for increased ITC area after DSAEK $(\beta=0.193-0.574$; all $P<0.02)$.

Conclusions: Higher preoperative total protein and specific cytokine levels in $\mathrm{AqH}$ were associated with ITC formation after DSAEK.

Translational Relevance: Our findings indicate that chronic pathological changes in AqH can cause PAS progression and glaucoma after DSAEK.

\section{Introduction}

Glaucoma is a serious complication after corneal transplantation that can lead to permanent loss of vision. The incidence of glaucoma varies among surgical procedures; for example, it has been calculated to be $9 \%$ to $35 \%$ after penetrating keratoplasty (PKP), ${ }^{1-4} 0 \%$ to $4.5 \%$ after anterior lamellar keratoplasty (ALK), ${ }^{5-7}$ and $2 \%$ to $14 \%$ after endothelial keratoplasty (EK). ${ }^{8-12}$
The trends of increases in postoperative transient intraocular pressure (IOP) also vary among these procedures: $29 \%$ to $80 \%$ after PKP, $17 \%$ to $36 \%$ after ALK, and $31 \%$ to $60 \%$ after EK. ${ }^{1-12}$ Various mechanisms are involved in elevated IOP, including intraoperative viscoelastic material, pupillary block due to air tamponade, response to topical steroids, damage to outflow mechanisms, and angle closure due to peripheral anterior synechiae (PAS). ${ }^{1,9}$ PAS is known to cause refractory glaucoma after corneal transplantation, ${ }^{13-15}$ 
Table 1. Patient Demographics

\begin{tabular}{lc} 
Demographic & Value \\
\hline Eyes, $n$ & 146 \\
Sex, $n(\%)$ & $61(42)$ \\
$\quad$ Male & $85(58)$ \\
Female & $72.9 \pm 10.5(27-95)$ \\
Age (yr), mean \pm SD (range) & $23.4 \pm 1.80$ \\
Axial length (mm), mean \pm SD & $1.7 \pm 1.5$ \\
Number of previous intraocular surgeries, mean \pm SD & 1.7 \\
\hline
\end{tabular}

iridocorneal endothelial syndrome, ${ }^{16,17}$ and uveitis. ${ }^{18}$ However, the exact mechanism of PAS development remains poorly understood.

Recent advances in anterior segment optical coherence tomography (AS-OCT) have enabled non-invasive and accurate quantification of iridotrabecular contact (ITC). ${ }^{13-15,19,20}$ Recently, we have shown that alterations in the aqueous humor $(\mathrm{AqH})$ microenvironment, such as elevated total protein and cytokine levels, significantly influence the long-term prognosis of corneal endothelial cells after PKP and Descemet stripping automated endothelial keratoplasty (DSAEK). ${ }^{21-23}$ Through these AqH investigations, we noticed that PAS developed after corneal transplantation in eyes with high $\mathrm{AqH}$ total protein and cytokine levels. Thus, we hypothesized that high $\mathrm{AqH}$ total protein and cytokine levels may be associated with PAS formation after DSAEK, leading to an increase in IOP and refractory glaucoma. In the current study, we first measured $\mathrm{AqH}$ protein and cytokine levels before DSAEK and analyzed ITC alterations after DSAEK using AS-OCT. Second, we evaluated the correlations between $\mathrm{AqH}$ total protein and cytokine levels and ITC alterations. Third, we evaluated the association between ITC and the incidence of IOP increase after DSAEK.

\section{Methods}

This prospective study adhered to the tenets of the Declaration of Helsinki. This study was approved by the institutional review board of Tokyo Dental College, Ichikawa General Hospital (I-15-42R). Written informed consent was obtained from all of the participants before the intervention.

\section{Participants}

A total of 146 consecutive patients who underwent DSAEK at Tokyo Dental College, Ichikawa General
Hospital, between November 2015 and March 2019 were included in this study (Table 1). The etiologies of DSAEK in the studied eyes included pseudophakic bullous keratopathy (PBK, 67 eyes), post-laser iridotomy BK (LI-BK, 25 eyes), Fuchs endothelial corneal dystrophy (FECD, 20 eyes), post-trabeculectomy BK (12 eyes), uveitis (five eyes), post-PKP endothelial decompensation (four eyes), BK due to birth injury (four eyes), and other causes (nine eyes).

\section{Surgical Technique}

DSAEK surgery was performed using the doubleglide technique. ${ }^{24,25}$ After sub-Tenon anesthesia using an injection of $2 \%$ lidocaine, a $5.0-\mathrm{mm}$ temporal corneoscleral incision was made. An anterior chamber maintenance cannula was inserted through the paracentesis at the 2 o'clock or 10 o'clock position, and Descemet stripping was performed using a reversebent Sinsky hook (ASICO, Westmont, IL). The recipient's endothelium and Descemet's membrane were carefully removed using forceps. Pre-cut donor grafts were trephinated, and the endothelial surface of the donor lenticle was coated with a small amount of viscoelastic material. Donor tissue was gently inserted into the anterior chamber using the Busin glide (ASICO). Air was carefully injected into the anterior chamber to unfold the graft. At 10 minutes after air injection, half of the air was replaced with a balanced salt solution (Alcon, Fort Worth, TX). At the end of the surgery, $2 \mathrm{mg}$ of subconjunctival betamethasone was administered. In patients with significant lens opacity (32 eyes), standard phacoemulsification and aspiration were performed with implantation of an intraocular lens, followed by DSAEK. Therefore, we performed solitary DSAEK in 111 eyes, DSAEK combined with simultaneous cataract surgery in 32 eyes, and DSAEK combined with simultaneous intraocular lens suture in three eyes. All DSAEK procedures were successful and uneventful, without the need for excessive intraoperative manipulation. 


\section{Postoperative Care}

After DSAEK, patients were prescribed topical eye drops of levofloxacin (Cravit; Santen, Osaka, Japan) and betamethasone $0.1 \%$ (Sanbetazon; Santen) five times a day. Topical betamethasone eye drops were tapered over the following 6 months. Starting at 6 months after DSAEK, we prescribed fluorometholone $0.1 \%$ eye drops (Flumetholon $0.1 \%$; Santen) three times a day for up to 12 months after surgery. Forty patients experienced mild IOP elevation $(\geq 21 \mathrm{mmHg}$ ), which resolved with cessation of topical steroids and/or topical antiglaucoma agents. There were no cases of graft rejection during the 12-month follow-up.

\section{Anterior Segment Optical Coherence Tomography Imaging}

All patients underwent AS-OCT examinations preoperatively and at 3, 6, and 12 months postoperatively. AS-OCT (CASIA Corneal/Anterior Segment OCT SS-1000; Tomey Corporation, Nagoya, Japan) is a type of Fourier-domain OCT that uses a $1320-\mathrm{nm}$ wavelength scanning laser source and a photodetector to detect wavelength-resolved interference signals. All eyes were imaged by trained technicians using an "angle analysis" protocol, which was composed of 128 radial B-scans, each with 512 A-scans (16-mm scan length). This allowed $360^{\circ}$ imaging of the whole anterior segment in 2.4 seconds. Eyes receiving topical medications that affected pupil size were not included.

\section{Measurement of the Area, Degree, and Maximum Height of the ITC}

To examine the ITC, the angle structure was analyzed using AS-OCT. The extent of ITC in each meridian was measured using the built-in software after manual detection of the scleral spur and anterior irido-angle adhesion (iris endpoint; Figs. 1A, 1B). The software automatically aligned the scleral spur (solid red line in the polar plot; Figs. 1C, 1D) and the iris endpoint (dotted green line in the polar plot) of individual cross-sectional OCT images and then computed the ITC area $\left(\mathrm{mm}^{2}\right)$, which was defined as the area bound below the iris endpoint and above the scleral spur (blue area in Figs. 1C, 1D). The
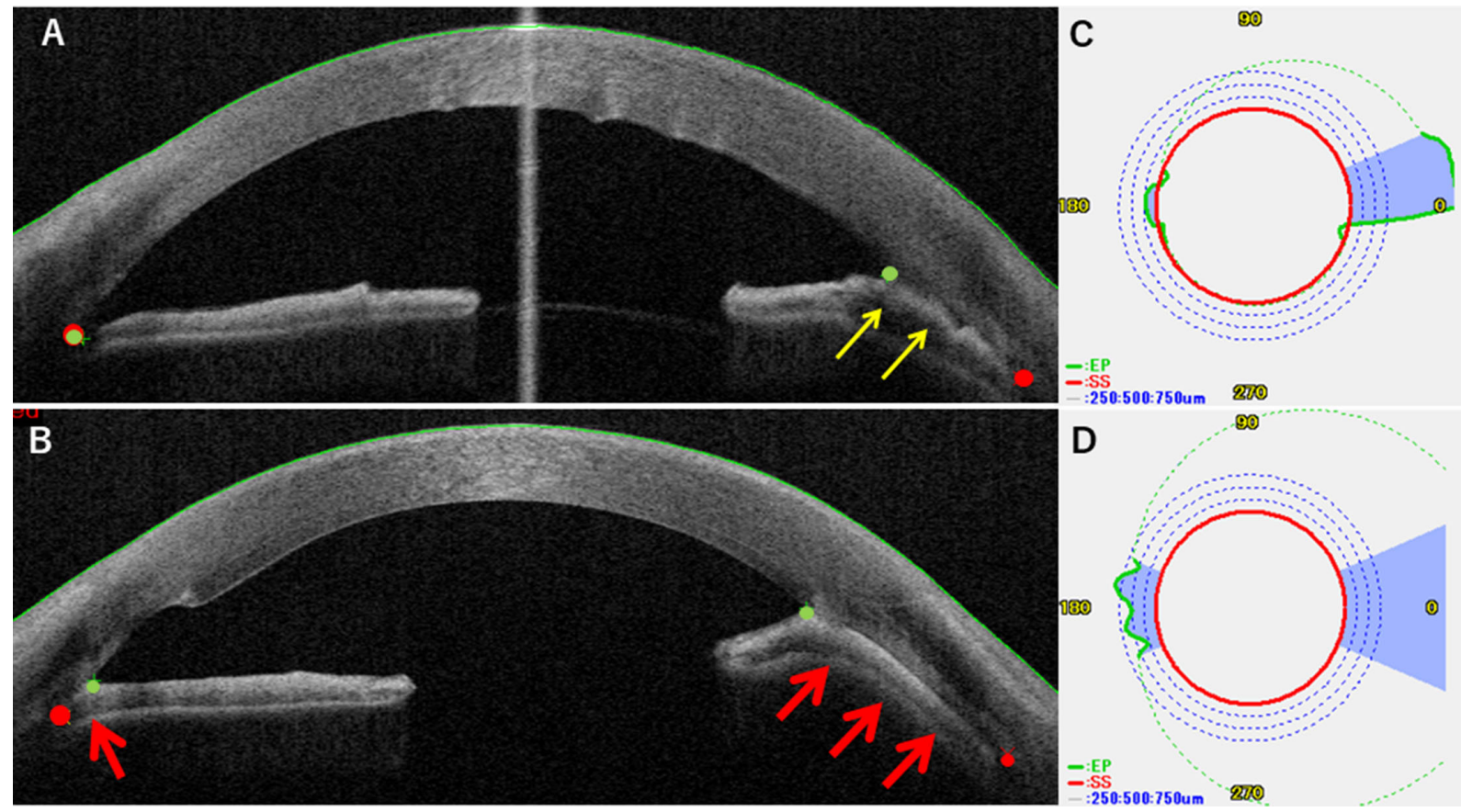

Figure 1. (A, B) Anterior segment OCT images of a representative patient before (A) and after (B) DSAEK. An 84-year-old man with LI-BK successfully underwent DSAEK. The total protein level in the aqueous humor was $3.46 \mathrm{mg} / \mathrm{mL}$. Before DSAEK, the patient had a limited ITC on the temporal side (A, yellow arrows). After DSAEK, ITC developed on the nasal side and expanded on the temporal side (B, red arrows). Red points represent the scleral spur, and green points indicate the peripheral endpoint of the iris. (C-D) ITC analysis using AS-OCT. The inbuilt software automatically aligns the scleral spur (red polar plot), the iris endpoint (green polar plot), and the ITC area (blue area) based on the individual cross-sectional OCT images. The software calculates the ITC degree, maximum ITC height $(\mathrm{mm})$, and ITC area (mm²). In this patient, the ITC degree increased from $65 \%$ to $100 \%$ after DSAEK. The maximum ITC height increased from $2.2 \mathrm{~mm}$ to $3.6 \mathrm{~mm}$ after DSAEK, and the ITC area increased from $7.53 \mathrm{~mm}^{2}$ to $20.94 \mathrm{~mm}^{2}$. The patient lost light sensation due to refractory glaucoma at 15 months after DSAEK. 

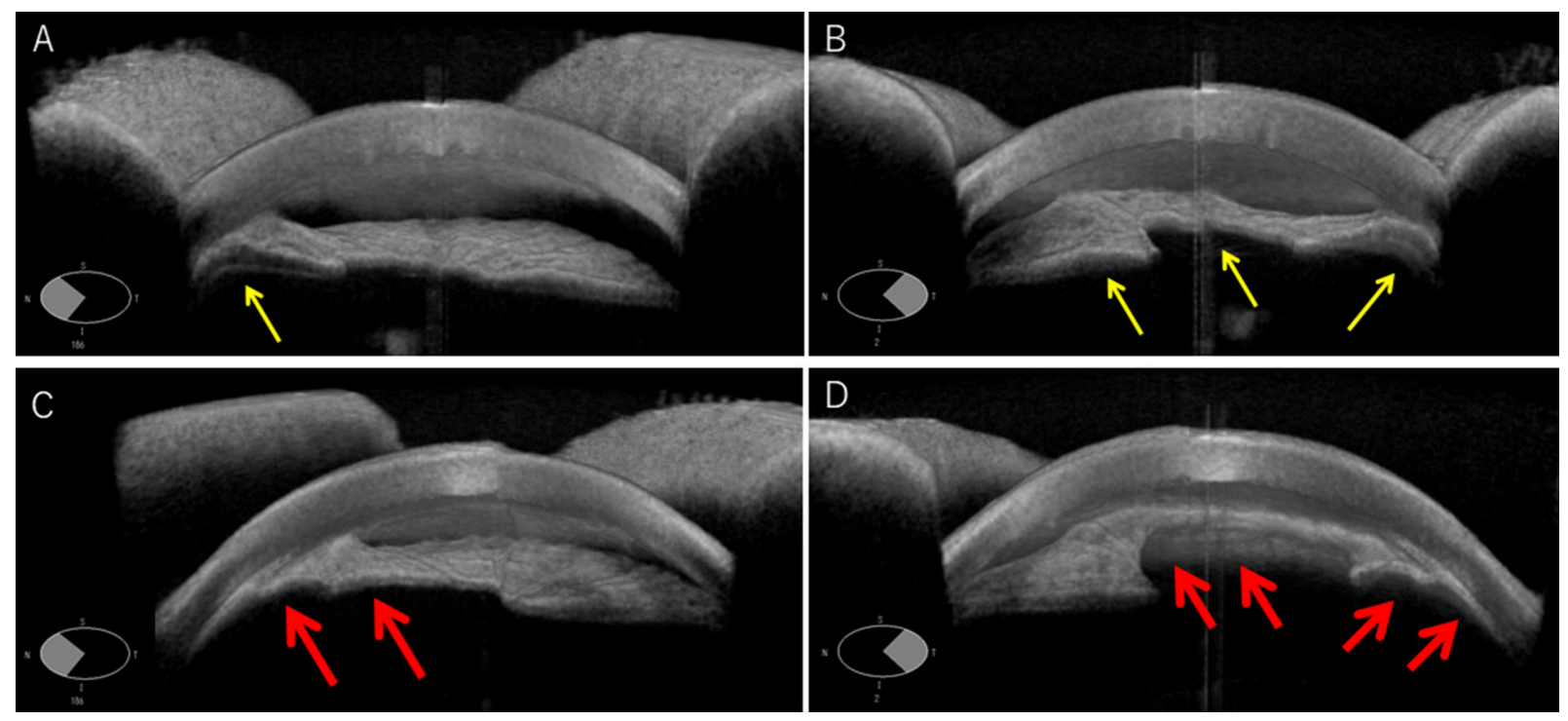

Figure 2. Three-dimensional images of ITC before DSAEK (A, nasal; B, temporal; yellow arrows) and after DSAEK (C, nasal; D, temporal; red arrows).

parameters were automatically calculated: (1) ITC $\%$ degree, the percentage of degrees that presented with ITC out of the total angle degrees analyzed; (2) maximum ITC height $(\mathrm{mm})$, the maximum distance from the scleral spur to the iris endpoint; and (3) ITC area $\left(\mathrm{mm}^{2}\right)$. In the present study, angle analysis could not be performed in the upper or lower areas, as the eyelids obstructed angle imaging in some patients. Therefore, the ITC was measured at a total range of $90^{\circ}$ on the nasal and temporal sides of the horizontal line, $45^{\circ}$ each, which were imaged at all time points in all patients (Fig. 2).

\section{Aqueous Humor Samples}

AqH samples containing 70 to $300 \mu \mathrm{L}$ were obtained under sterile conditions at the beginning of surgery after topical anesthesia in DSAEK surgery. First, paracentesis was performed on the clear cornea. $\mathrm{AqH}$ samples were obtained using a 27 -gauge needle, taking care not to touch the iris, lens, or corneal endothelium. The samples were centrifuged at $3000 \mathrm{~g}$ for 5 minutes. The soluble fractions were collected and stored at $-80^{\circ} \mathrm{C}$.

\section{Total Protein and Cytokine Level Measurements}

The total protein levels of the $\mathrm{AqH}$ samples were determined using a DC Protein Assay (Bio-Rad, Hercules, CA). ${ }^{26}$ In brief, bovine serum albumin (BSA) was used as a standard in the range of 0.06 to
$1.37 \mathrm{mg} / \mathrm{mL}$. Samples $(5 \mu \mathrm{L})$ of BSA and $\mathrm{AqH}$ were added to 96-well microplates, followed by the immediate addition of a mixture containing $25 \mu \mathrm{L}$ reagent $\mathrm{A}$ and $200 \mu \mathrm{L}$ reagent $\mathrm{C}$. After 15 minutes of incubation at room temperature in the dark, the microplates were read at $690 \mathrm{~nm}$ and 405 $\mathrm{nm}$ using a microplate reader (Model 550; BioRad). The cytokine levels of interleukin (IL)- $1 \alpha$, IL-1 $\beta$, IL-4, IL-6, IL-8, IL-10, IL-12p70, IL-13, IL-17A, macrophage inflammatory protein (MIP)$1 \alpha$, MIP-1 $\beta$, monocyte chemotactic protein (MCP)1 , tumor necrosis factor (TNF)- $\alpha$, interferon (IFN)$\alpha$, IFN- $\gamma$, E-selectin, P-selectin, soluble intercellular adhesion molecule (sICAM)-1, interferon gammainduced protein (IP)-10, and granulocyte-macrophage colony-stimulating factor (GM-CSF) in $\mathrm{AqH}$ samples were measured using a Luminex bead-based multiplex immunoassay (ProcaPlex kit; Luminex, San Antonio, TX) according to a previous report. ${ }^{27}$ Briefly, $50 \mu \mathrm{L}$ of AqH samples were incubated with antibody-coated capture beads in an incubation buffer at room temperature. After a 2-hour incubation, the beads were washed three times using washing buffer, and phycoerythrinlabeled streptavidin was added for 30 minutes in the dark at room temperature. After washing three times with washing buffer, the plates were resuspended in 150 $\mu \mathrm{L}$ of reading buffer, and the assays were performed using a Luminex 200 Multiplexing Instrument.

\section{Statistical Analysis}

Data were analyzed using Stata/IC 14.0 for iOS (StataCorp, College Station, TX). The Shapiro-Wilk 
Aqueous Cytokine and Peripheral Anterior Synechiae

test was used to assess whether the data were normally distributed. Spearman's correlation analyses were used to evaluate the correlations between the total protein and cytokine levels in the AqH and ITC \%degree, maximum height, and area. Fisher's exact test was used to assess the differences in the incidence of postDSAEK IOP rise $(\geq 21 \mathrm{mmHg})$ between the groups with and without ITC. For multivariate analyses of the clinical factors that were correlated with the progression of ITC area, we selected five independent clinical factors (preoperative total protein levels in $\mathrm{AqH}$, preoperative ITC, axial length, graft size, and age; variance inflation factor $=1.11-1.12$ ) and conducted multiple linear regression analyses. Data are expressed as mean \pm standard deviation (SD). Statistical significance was set at $P<0.05$.

\section{Results}

\section{Clinical Results}

All of the DSAEK surgeries were successful. The logarithm of minimal angle resolution (logMAR) significantly improved from $1.30 \pm 0.71$ preoperatively to $0.45 \pm 0.40$ at 3 months, $0.38 \pm 0.39$ at 6 months, and $0.36 \pm 0.46$ at 12 months (all $P<$ $0.0001)$. Corneal endothelial cell density $\left(\right.$ cells $\left./ \mathrm{mm}^{2}\right)$ of the graft decreased from $2746 \pm 249$ to $1566 \pm 592$ at 3 months, $1433 \pm 556$ at 6 months, and $1253 \pm 600$ at 12 months (all $P<0.0001$ ). Postoperative complications that occurred included pupillary block due to air tamponade in three eyes $(2 \%)$ and graft detachment requiring air injection in five eyes (4\%), all of which were treated appropriately.

\section{ITC Indices}

ITC developed in 10 eyes $(6.8 \%)$ without preoperative ITC and progressed in 22 eyes $(15.1 \%)$ with preoperative ITC; it did not progress in 15 eyes $(10.3 \%)$ with preoperative ITC. The ITC indices (ITC \%degree, maximal height, and area) in 146 eyes increased after
TVST | September 2021 | Vol. 10 | No. 11 | Article 12 | 5

DSAEK; however, there were no statistically significant differences compared with the preoperative values (all $P \geq 0.11)$ (Table 2).

\section{Correlation Between Total Protein Level in AqH and ITC Indices}

The preoperative total protein level of the $\mathrm{AqH}$ was $1.14 \pm 0.77 \mathrm{mg} / \mathrm{dL}$. The mean protein level was significantly higher in 47 eyes with ITC or ITC progression than in those without $(P=0.04)$. The preoperative total protein levels in the AqH were significantly correlated with the preoperative ITC \%degree and maximum height (Table 3; all $P \leq 0.026$ ). Furthermore, the preoperative total protein levels in the $\mathrm{AqH}$ were significantly correlated with the ITC \%degree, maximum height, and area after DSAEK (all $P \leq 0.0002$ ). The preoperative total protein levels in the $\mathrm{AqH}$ were significantly positively correlated with $\Delta \%$ degree, $\Delta$ maximum height, and $\Delta$ area (all $P \leq 0.033$ ).

\section{Multivariate Analysis}

Although these data indicated an association between total protein levels in the $\mathrm{AqH}$ and ITC progression after DSAEK, other clinical factors, such as the presence of preoperative ITC, larger grafts, and shorter axial length (shallow anterior chamber), can also be potential confounding factors. Thus, to identify the risk factors for the progression of ITC, we conducted a multivariate analysis. We selected the $\triangle$ ITC area, as it represents a three-dimensional alteration of ITC (\%degree and maximum height are twodimensional parameters) and had the greatest standard correlation coefficients in the correlation analyses with the total protein levels in the AqH. Multivariate analyses showed that the total protein levels in the $\mathrm{AqH}$ and the presence of preoperative ITC factors were significantly associated with the progression of ITC area $(\beta=$ 0.193-0.574; all $P<0.020$ ), whereas axial length, graft size, and patient age were not (Table 4).

\section{Table 2. Time Course Alteration in ITC Indices}

\begin{tabular}{lcccc} 
& \multicolumn{4}{c}{ Mean \pm SD $\left(P^{\mathrm{a}}\right)$} \\
\cline { 2 - 5 } ITC & Preop & $3 \mathrm{mo}$ & $6 \mathrm{mo}$ & $12 \mathrm{mo}$ \\
\hline \%Degree & $17.8 \pm 32.1$ & $18.8 \pm 31.2(0.89)$ & $20.2 \pm 34.1(0.45)$ & $22.6 \pm 33.6(0.33)$ \\
Maximal height $(\mathrm{mm})$ & $0.46 \pm 0.90$ & $0.51 \pm 0.92(0.11)$ & $0.53 \pm 1.00(0.12)$ & $0.58 \pm 1.05(0.17)$ \\
Area $\left(\mathrm{mm}^{2}\right)$ & $2.00 \pm 4.42$ & $2.10 \pm 4.86(0.13)$ & $2.71 \pm 6.47(0.16)$ & $3.00 \pm 6.85(0.17)$ \\
\hline
\end{tabular}

${ }^{a} P$ values compared with preoperative values ( $n=146$ eyes). 
Table 3. Correlations Between Total Protein Levels in AqH and ITC Indices

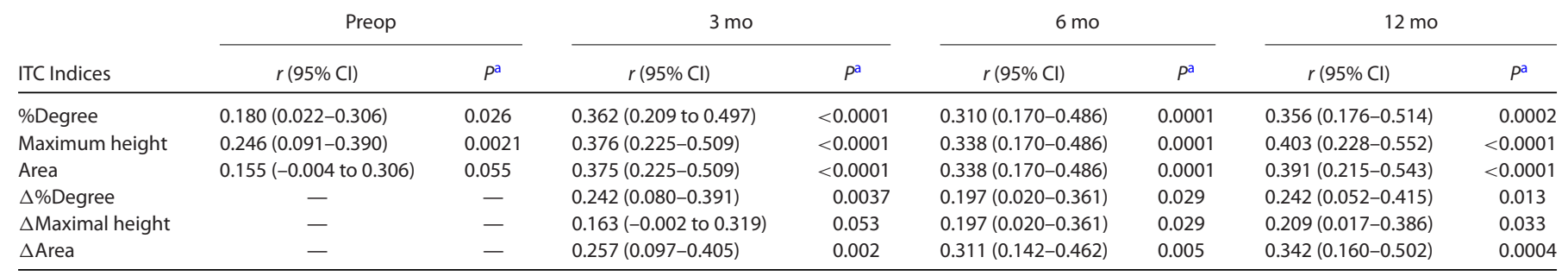

$\Delta \%$ Degree $=$ (postoperative ITC degree) - (preoperative ITC degree). $\Delta$ Maximal height $=$ (postoperative ITC maximal height) - (preoperative ITC maximal height). $\Delta$ Area = (postoperative ITC area) - (preoperative ITC area).

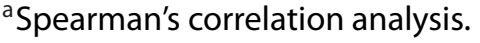

Table 4. Multivariate Analysis for Clinical Factors Associated With Progression of ITC Area

\begin{tabular}{|c|c|c|c|c|c|c|}
\hline \multirow[b]{2}{*}{$\Delta$ Area } & \multicolumn{2}{|c|}{$3 \mathrm{mo}$} & \multicolumn{2}{|c|}{$6 \mathrm{mo}$} & \multicolumn{2}{|c|}{$12 \mathrm{mo}$} \\
\hline & $\beta$ & $P$ & $\beta$ & $P$ & $\beta$ & $P$ \\
\hline Preop total protein levels & 0.221 & 0.002 & 0.226 & 0.003 & 0.193 & 0.020 \\
\hline Preop ITC $(0 \text { or } 1)^{\mathrm{a}}$ & 0.551 & 0.000 & 0.574 & 0.000 & 0.574 & 0.000 \\
\hline Axial length (mm) & -0.003 & 0.967 & -0.002 & 0.976 & 0.031 & 0.704 \\
\hline Graft size (mm) & -0.054 & 0.444 & -0.064 & 0.410 & -0.033 & 0.679 \\
\hline Age & -0.068 & 0.333 & -0.100 & 0.191 & -0.073 & 0.365 \\
\hline Adjusted $R^{2}$ & \multicolumn{2}{|c|}{0.443} & \multicolumn{2}{|c|}{0.454} & \multicolumn{2}{|c|}{0.452} \\
\hline
\end{tabular}

$\Delta$ Area $=$ (postoperative ITC area) - (preoperative ITC area). Variance inflation factor $=1.11$ to 1.12.

apresence of preoperative ITC was dichotomized as categorical variables for multivariate regression analysis as follows: absence $=0$, presence $=1$.

Table 5. Association Between Presence of ITC and Increase in IOP After DSAEK

\section{Association Between ITC and Intraocular Pressure Increase}

At 12 months, ITC was successfully observed in 96 of the 146 eyes through the use of AS-OCT. Among these 96 eyes, an IOP increase greater than $21 \mathrm{mmHg}$ was observed in 14 of 29 eyes with ITC (48.3\%) and in 15 of 65 eyes without ITC $(23.1 \%)$. The incidence of IOP increase greater than $21 \mathrm{mmHg}$ was significantly higher in eyes with ITC than in those without ITC ( $P$ $=0.026)$ (Table 5).

\section{Cytokine Levels in Eyes With and Without ITC}

We showed that the preoperative protein level in $\mathrm{AqH}$ is a relevant factor for ITC formation after DSAEK; however, the exact reasons remain elusive. Therefore, we hypothesized that inflammation in the $\mathrm{AqH}$ plays a pivotal role in ITC formation, leading to an IOP increase after DSAEK. We measured and compared the inflammatory cytokine levels in the $\mathrm{AqH}$ between the eyes with and without the presence of ITC after DSAEK (Table 6). The levels of IL-6, IL-8, IL17A, MCP-1, IFN- $\gamma$, E-selectin, and sICAM-1 were significantly higher in 47 eyes with ITC or ITC progression than in those without $(P \leq 0.045)$.

\section{Correlation Between Cytokine Level in Aqueous Humor and ITC Indices}

To substantiate these results, we further evaluated the correlations between cytokine levels in the $\mathrm{AqH}$ and ITC areas (Table 7). We selected the ITC area for the same reason as mentioned earlier. The results showed that the preoperative ITC area was significantly positively correlated with preoperative levels of IL-8, IL-17A, IFN- $\gamma$, E-selectin, and sICAM-1 $(P \leq 0.005)$. 
Table 6. Preoperative Aqueous Total Protein/Cytokine Levels Stratified by Presence of PAS After DSAEK

\begin{tabular}{|c|c|c|c|}
\hline & \multicolumn{3}{|c|}{ Mean \pm SD } \\
\hline & $\operatorname{PAS}(-)(n=99)$ & $\operatorname{PAS}(+)(n=47)$ & $P^{a}$ \\
\hline Total protein $(\mathrm{mg} / \mathrm{mL})$ & $1.00 \pm 0.57$ & $1.45 \pm 1.03$ & 0.036 \\
\hline $\mathrm{IL}-1 \alpha(\mathrm{pg} / \mathrm{mL})$ & $46.1 \pm 65.4$ & $51.3 \pm 93.1$ & 0.926 \\
\hline $\mathrm{IL}-1 \beta(\mathrm{pg} / \mathrm{mL})$ & $3.51 \pm 6.37$ & $4.79 \pm 6.48$ & 0.095 \\
\hline IL-4 (pg/mL) & $31.8 \pm 50.3$ & $43.7 \pm 64.7$ & 0.815 \\
\hline IL-6 (pg/mL) & $947 \pm 1620$ & $1270 \pm 1150$ & 0.043 \\
\hline IL-8 (pg/mL) & $64.7 \pm 93.3$ & $122 \pm 149$ & 0.003 \\
\hline IL-10 (pg/mL) & $5.91 \pm 14.4$ & $10.2 \pm 29.6$ & 0.148 \\
\hline IL-12p70 (pg/mL) & $12.1 \pm 7.56$ & $20.8 \pm 17.0$ & 0.100 \\
\hline IL-13 (pg/mL) & $8.05 \pm 4.28$ & $7.99 \pm 4.92$ & 0.711 \\
\hline IL-17A (pg/mL) & $11.1 \pm 14.7$ & $20.8 \pm 14.3$ & 0.002 \\
\hline MIP-1 $\alpha(\mathrm{pg} / \mathrm{mL})$ & $32.5 \pm 80.2$ & $21.1 \pm 33.8$ & 0.203 \\
\hline MIP-1 $\beta(\mathrm{pg} / \mathrm{mL})$ & $130 \pm 202$ & $90.8 \pm 154$ & 0.740 \\
\hline MCP-1 (pg/mL) & $913 \pm 612$ & $1120 \pm 643$ & 0.045 \\
\hline TNF- $\alpha$ (pg/mL) & $59.2 \pm 62.3$ & $56.0 \pm 65.5$ & 0.985 \\
\hline IFN- $\alpha(p g / m L)$ & $2.71 \pm 2.54$ & $1.96 \pm 2.92$ & 0.228 \\
\hline IFN- $\gamma(p g / m L)$ & $110 \pm 161$ & $238 \pm 184$ & $<0.001$ \\
\hline E-selectin (pg/mL) & $3720 \pm 2290$ & $7000 \pm 6620$ & 0.015 \\
\hline P-selectin (pg/mL) & $6970 \pm 5570$ & $12300 \pm 1700$ & 0.161 \\
\hline sICAM-1 (pg/mL) & $3340 \pm 3970$ & $7440 \pm 5770$ & $<0.001$ \\
\hline IP-10 (pg/mL) & $236 \pm 340$ & $136 \pm 123$ & 0.220 \\
\hline GM-CSF (pg/mL) & $15.3 \pm 13.7$ & $14.9 \pm 8.25$ & 0.706 \\
\hline
\end{tabular}

${ }^{a}$ Mann-Whitney $U$ test, compared between eyes with and without PAS after DSAEK.

Furthermore, the ITC area at 3 months was significantly correlated with the preoperative $\mathrm{AqH}$ levels of IL-8, IL-17A, IFN- $\gamma$, E-selectin, and sICAM-1 $(P \leq$ $0.015)$. The ITC area at 6 months was significantly correlated with the preoperative AqH levels of IL-1 $\beta$, IL-8, IL-17A, IFN- $\gamma$, E-selectin, and sICAM-1 $(P \leq$ $0.050)$. The ITC area at 12 months was significantly correlated with the preoperative AqH levels of IL-8, IFN- $\gamma$, and sICAM-1 $(P \leq 0.021)$.

\section{Discussion}

Glaucoma is a serious complication of corneal transplantation. The visual outcome significantly worsens in patients with post-keratoplasty glaucoma due to unrecoverable loss of the visual field and increased risk of graft failure. ${ }^{28}$ The development of post-keratoplasty glaucoma has been reported to be associated with trauma, inflammation, and aphakic BK. ${ }^{29}$ Previous studies have indicated that AS-OCT is useful for assessing PAS and angle structure in cases with opaque corneas, where details regarding the PAS are not visible. ${ }^{13-15,19,20}$ The incidence of PAS varied from $11 \%$ to $56 \%$, presumably due to different etiologies. ${ }^{15,30}$ Maier et al..$^{30}$ evaluated PAS using AS-OCT and reported that PAS was significantly associated with the development of post-keratoplasty glaucoma after both PKP and DSAEK.

Glaucoma due to PAS formation does not seem to be a common complication after DSAEK, ${ }^{31}$ compared to PKP, particularly in Western countries where FECD is the major etiology of endothelial keratoplasty. ${ }^{32}$ In contrast, in Asian countries, where complicated PBK or LI-BK is the cause of $60 \%$ to $70 \%$ of BK cases, ${ }^{33,34}$ we observed PAS formation and progression in some patients after DSAEK. However, in a recent study conducted in New York, which included 353 DSAEK procedures (FECD in $40 \%, \mathrm{PBK} /$ aphakic BK in $40 \%$, failed PKP in $13.3 \%$, and post-glaucoma surgery in $6.7 \%)$, Wu et al. ${ }^{31}$ reported that PAS occurred in 37 patients after DSAEK (10.5\%), although AS-OCT was not used for PAS evaluation. Furthermore, a previous report from Germany showed that the rise in IOP after DSAEK was significantly greater in PBK than in FECD,${ }^{30}$ although the authors did not compare PAS between patients with PBK and those with FECD. Thus, we believe that PAS formation and rise in IOP 
Table 7. Correlations Between Preoperative Cytokine Levels and PAS Area After DSAEK

\begin{tabular}{|c|c|c|c|c|c|c|c|c|}
\hline & \multicolumn{8}{|c|}{ PAS Area } \\
\hline & \multicolumn{2}{|c|}{ Preop } & \multicolumn{2}{|c|}{$3 \mathrm{mo}$} & \multicolumn{2}{|c|}{$6 \mathrm{mo}$} & \multicolumn{2}{|c|}{$12 \mathrm{mo}$} \\
\hline & $r$ & $p^{a}$ & $r$ & $p^{a}$ & $r$ & $p^{a}$ & $r$ & $P^{a}$ \\
\hline $\mathrm{IL}-1 \alpha(\mathrm{pg} / \mathrm{mL})$ & 0.0002 & 0.999 & 0.008 & 0.938 & 0.031 & 0.782 & -0.012 & 0.914 \\
\hline $\mathrm{IL}-1 \beta(\mathrm{pg} / \mathrm{mL})$ & 0.220 & 0.104 & 0.258 & 0.062 & 0.291 & 0.050 & 0.182 & 0.221 \\
\hline IL-4 (pg/mL) & 0.115 & 0.259 & 0.046 & 0.657 & 0.081 & 0.494 & 0.018 & 0.876 \\
\hline IL-6 (pg/mL) & 0.121 & 0.198 & 0.198 & 0.037 & 0.231 & 0.029 & 0.186 & 0.078 \\
\hline IL-8 (pg/mL) & 0.314 & 0.001 & 0.282 & 0.004 & 0.350 & 0.001 & 0.252 & 0.021 \\
\hline IL-10 (pg/mL) & 0.104 & 0.340 & 0.173 & 0.121 & 0.162 & 0.190 & 0.211 & 0.085 \\
\hline IL-12p70 (pg/mL) & 0.230 & 0.097 & 0.169 & 0.236 & 0.282 & 0.070 & 0.170 & 0.281 \\
\hline IL-13 (pg/mL) & 0.173 & 0.353 & 0.061 & 0.749 & 0.324 & 0.123 & 0.031 & 0.879 \\
\hline IL-17A (pg/mL) & 0.409 & 0.005 & 0.410 & 0.006 & 0.430 & 0.009 & 0.318 & 0.055 \\
\hline MIP-1 $\alpha(\mathrm{pg} / \mathrm{mL})$ & -0.146 & 0.275 & -0.187 & 0.171 & -0.186 & 0.222 & -0.210 & 0.166 \\
\hline MIP-1 $\beta(\mathrm{pg} / \mathrm{mL})$ & 0.019 & 0.840 & -0.006 & 0.947 & 0.026 & 0.808 & -0.054 & 0.616 \\
\hline MCP-1 (pg/mL) & 0.146 & 0.111 & 0.151 & 0.106 & 0.190 & 0.065 & 0.087 & 0.397 \\
\hline TNF- $\alpha(\mathrm{pg} / \mathrm{mL})$ & 0.107 & 0.395 & -0.007 & 0.959 & -0.028 & 0.845 & -0.172 & 0.223 \\
\hline IFN- $\alpha(\mathrm{pg} / \mathrm{mL})$ & 0.107 & 0.395 & -0.148 & 0.316 & -0.231 & 0.141 & -0.233 & 0.147 \\
\hline $\mathrm{IFN}-\gamma(\mathrm{pg} / \mathrm{mL})$ & 0.448 & 0.0003 & 0.445 & 0.0004 & 0.466 & 0.0007 & 0.318 & 0.009 \\
\hline E-selectin (pg/mL) & 0.326 & 0.002 & 0.265 & 0.015 & 0.299 & 0.013 & 0.197 & 0.096 \\
\hline P-selectin (pg/mL) & 0.151 & 0.100 & 0.126 & 0.178 & 0.082 & 0.429 & 0.023 & 0.825 \\
\hline sICAM-1 (pg/mL) & 0.308 & 0.0007 & 0.336 & 0.0003 & 0.304 & 0.003 & 0.292 & 0.004 \\
\hline $\mathrm{IP}-10(\mathrm{pg} / \mathrm{mL})$ & -0.095 & 0.303 & -0.089 & 0.344 & -0.073 & 0.477 & -0.087 & 0.397 \\
\hline GM-CSF (pg/mL) & 0.057 & 0.829 & -0.032 & 0.905 & -0.093 & 0.732 & 0.055 & 0.856 \\
\hline
\end{tabular}

aspearman's correlation analysis.

after DSAEK are clinically relevant issues in both Western and Asian countries.

Clinically, PAS is associated with chronic inflammation in the anterior chamber, a history of narrow angle, and breakdown of the blood-aqueous barrier $(\mathrm{BAB})^{35}$; however, the mechanism of PAS formation remains elusive. Previous studies have shown that panretinal photocoagulation ${ }^{36}$ and diode laser trabeculoplasty ${ }^{37}$ caused temporal increases in flare photon levels; however, they did not cause PAS formation. In the current study, PAS formation 1 year after DSAEK was significantly correlated with high protein levels in the AqH. The high total protein levels in the $\mathrm{AqH}$ can reflect a chronic breakdown of the $\mathrm{BAB}$ due to iris damage in BK eyes. ${ }^{26,27}$

In the current study, MCP-1 and IL-6 levels were associated with the presence of PAS, but not with PAS area at any time point. In contrast, the levels of IL8, IL-17A, IFN- $\gamma$, and sICAM-1 were associated with both the presence of PAS and PAS area. As there were complicated correlations among cytokine levels (Supplementary Table), we should be careful not to overinterpret the results; the elevated total protein and cytokine levels measured in the current study may not be the direct cause of PAS formation. The results of the current study suggest that a specific mechanism is involved in PAS formation after corneal transplantation that leads to glaucoma. We suggest two potential measures we can take to avoid refractory glaucoma due to PAS formation after corneal transplantation in the future: prevention and therapy. Minimizing iris damage during surgery could potentially prevent PAS formation, as it is known that iris damage can directly cause chronic elevation of cytokine levels in the AqH. To identify therapeutic molecules that can prevent PAS formation, proteomics is considered to be a powerful tool that can measure the level of more than 200 to 1000 proteins in the $\mathrm{AqH} .^{23,38}$ Further studies are required to elucidate the exact mechanism of PAS formation and IOP rise after corneal transplantation. The use of proteomic analysis of the $\mathrm{AqH}$ and transcriptomics analysis of the trabecular meshwork and peripheral iris tissues to identify alterations in the aqueous environment and the reaction of trabecular meshwork and peripheral iris tissues against it would be beneficial to understanding this mechanism.

This study had some limitations. First, the different graft sizes $(7.75-8.5 \mathrm{~mm})$ may have had some effect 


\section{Aqueous Cytokine and Peripheral Anterior Synechiae}

on PAS formation, as previously reported ${ }^{30}$ However, multivariate analyses showed that the different graft sizes did not correlate with the PAS parameters after DSAEK. Thus, we consider its influence to be minimal. Second, this study included heterogeneous etiologies for BK. Therefore, we conducted a subgroup analysis including only PBK, which showed similar results.

In conclusion, we evaluated PAS using AS-OCT and identified PAS in 47 eyes after DSAEK $(32.2 \%)$. We also showed that preoperative total protein levels were significantly correlated with PAS formation and progression after DSAEK. Furthermore, elevated levels of specific cytokines, such as IL-8, IL-17A, IFN$\gamma$, E-selectin, and sICAM-1 were significantly correlated with PAS formation after DSAEK. Additionally, MCP-1 and IL-6 levels were significantly higher in eyes with PAS than in those without PAS. These results suggest that microenvironmental changes in $\mathrm{AqH}$ cause progression of PAS as a result of chronic inflammation with elevated levels of specific cytokines.

\section{Acknowledgments}

The authors thank Editage for the English language editing.

Supported by a Grant-in-Aid for Scientific Research (15K10906 to TY) from the Ministry of Education, Culture, Sports, Science and Technology. The funding organization had no role in the design or conduct of this research.

Presented at the Japanese Cornea Conference, February 17, 2021.

Disclosure: Y. Kusano, None; T. Yamaguchi, None; S. Nishisako, None; T. Matsumura, None; M. Fukui, None; K. Higa, None; T. Inoue, None; J. Shimazaki, None

\section{References}

1. Goldberg DB, Schanzlin DJ, Brown SI. Incidence of increased intraocular pressure after keratoplasty. Am J Ophthalmol. 1981;92:372-377.

2. Karesh JW, Nirankari VS. Factors associated with glaucoma after penetrating keratoplasty. $A m J$ Ophthalmol. 1983;96:160-164.

3. Chien AM, Schmidt CM, Cohen EJ, et al. Glaucoma in the immediate postoperative period after penetrating keratoplasty. Am J Ophthalmol. 1993;115:711-714.
TVST | September 2021 | Vol. 10 | No. 11 | Article 12 | 9

4. Foulks GN. Glaucoma associated with penetrating keratoplasty. Ophthalmology. 1987;94:871-874.

5. Ogawa A, Yamaguchi T, Mitamura H, et al. Aetiology-specific comparison of long-term outcome of deep anterior lamellar keratoplasty for corneal diseases. Br J Ophthalmol. 2016;100:11761182.

6. Huang OS, Mehta JS, Htoon HM, Tan DT, Wong TT. Incidence and risk factors of elevated intraocular pressure following deep anterior lamellar keratoplasty. Am J Ophthalmol. 2016;170:153160.

7. Musa FU, Patil S, Rafiq O, Galloway P, Ball J, Morrell A. Long-term risk of intraocular pressure elevation and glaucoma escalation after deep anterior lamellar keratoplasty. Clin Exp Ophthalmol. 2012;40:780-785.

8. Vajaranant TS, Price MO, Price FW, Gao W, Wilensky JT, Edward DP. Visual acuity and intraocular pressure after Descemet's stripping endothelial keratoplasty in eyes with and without preexisting glaucoma. Ophthalmology. 2009;116:1644-1650.

9. Maier AK, Klamann MK, Torun N, et al. Intraocular pressure elevation and post-DSEK glaucoma after Descemet's stripping endothelial keratoplasty. Graefes Arch Clin Exp Ophthalmol. 2013;251:1191-1198.

10. Ozeki N, Yuki K, Shiba D, Shimmura S, Murat D, Tsubota K. Intraocular pressure elevation after Descemet's stripping endothelial keratoplasty. Jpn J Ophthalmol. 2012;56:307-311.

11. Maier AK, Wolf $\mathrm{T}$, Gundlach $\mathrm{E}$, et al. Intraocular pressure elevation and post-DMEK glaucoma following Descemet membrane endothelial keratoplasty. Graefes Arch Clin Exp Ophthalmol. 2014;252:1947-1954.

12. Muller L, Kaufmann C, Bachmann LM, Tarantino-Scherrer JN, Thiel MA, Bochmann F. Changes in intraocular pressure after Descemet stripping automated endothelial keratoplasty: a retrospective analysis. Cornea. 2015;34:271-274.

13. Karadag O, Kugu S, Erdogan G, Kandemir B, Eraslan Ozdil S, Dogan OK. Incidence of and risk factors for increased intraocular pressure after penetrating keratoplasty. Cornea. 2010;29:278282.

14. Memarzadeh F, Li Y, Francis BA, Smith RE, Gutmark J, Huang D. Optical coherence tomography of the anterior segment in secondary glaucoma with corneal opacity after penetrating keratoplasty. Br J Ophthalmol. 2007;91:189-192.

15. Fukuda R, Usui T, Tomidokoro A, et al. Noninvasive observations of peripheral angle in eyes 
Aqueous Cytokine and Peripheral Anterior Synechiae

after penetrating keratoplasty using anterior segment Fourier-domain optical coherence tomography. Cornea. 2012;31:259-263.

16. Teekhasaenee C, Ritch R. Iridocorneal endothelial syndrome in Thai patients: clinical variations. Arch Ophthalmol. 2000;118:187-192.

17. Silva L, Najafi A, Suwan Y, Teekhasaenee C, Ritch $\mathrm{R}$. The iridocorneal endothelial syndrome. Surv Ophthalmol. 2018;63:665-676.

18. Daniel E, Pistilli M, Kothari S, et al. Risk of ocular hypertension in adults with noninfectious uveitis. Ophthalmology. 2017;124:1196-1208.

19. Lim LS, Aung T, Husain R, Wu YJ, Gazzard G, Seah SK. Acute primary angle closure: configuration of the drainage angle in the first year after laser peripheral iridotomy. Ophthalmology. 2004;111:1470-1474.

20. Sanchez-Parra L, Pardhan S, Buckley RJ, Parker M, Bourne RR. Diurnal intraocular pressure and the relationship with swept-source OCT-derived anterior chamber dimensions in angle closure: the IMPACT study. Invest Ophthalmol Vis Sci. 2015;56:2943-2949.

21. Yazu H, Yamaguchi T, Aketa N, et al. Preoperative aqueous cytokine levels are associated with endothelial cell loss after Descemet's stripping automated endothelial keratoplasty. Invest Ophthalmol Vis Sci. 2018;59:612-620.

22. Yagi-Yaguchi $Y$, Yamaguchi $T$, Higa $K$, et al. Preoperative aqueous cytokine levels are associated with a rapid reduction in endothelial cells after penetrating keratoplasty. Am J Ophthalmol. 2017;181:166-173.

23. Yamaguchi $T$, Higa $K$, Yagi-Yaguchi $Y$, et al. Pathological processes in aqueous humor due to iris atrophy predispose to early corneal graft failure in humans and mice. Sci Adv. 2020;6:eaaz5195.

24. Yamaguchi T, Negishi K, Yamaguchi K, et al. Effect of anterior and posterior corneal surface irregularity on vision after Descemet-stripping endothelial keratoplasty. J Cataract Refract Surg. 2009;35:688-694.

25. Yamaguchi T, Ohnuma K, Tomida D, et al. The contribution of the posterior surface to the corneal aberrations in eyes after keratoplasty. Invest Ophthalmol Vis Sci. 2011;52:6222-6229.

26. Yamaguchi T, Higa K, Suzuki T, et al. Elevated cytokine levels in the aqueous humor of eyes with bullous keratopathy and low endothelial cell density. Invest Ophthalmol Vis Sci. 2016;57:59545962.
TVST | September 2021 | Vol. 10 | No. 11 | Article 12 | 10

27. Aketa N, Yamaguchi $\mathrm{T}$, Suzuki $\mathrm{T}$, et al. Iris damage is associated with elevated cytokine levels in aqueous humor. Invest Ophthalmol Vis Sci. 2017;58:Bio42-Bio51.

28. Irvine AR, Kaufman HE. Intraolar pressure following penetrating keratoplasty. Am J Ophthalmol. 1969;68:835-844.

29. Ayyala RS. Penetrating keratoplasty and glaucoma. Surv Ophthalmol. 2000;45:91-105.

30. Maier AK, Gundlach E, Gonnermann J, et al. Anterior segment analysis and intraocular pressure elevation after penetrating keratoplasty and posterior lamellar endothelial keratoplasty. Ophthalmic Res. 2015;53:36-47.

31. Wu EI, Ritterband DC, Yu G, Shields RA, Seedor JA. Graft rejection following Descemet stripping automated endothelial keratoplasty: features, risk factors, and outcomes. Am J Ophthalmol. 2012;153:949-957.e1.

32. Price MO, Gorovoy M, Benetz BA, et al. Descemet's stripping automated endothelial keratoplasty outcomes compared with penetrating keratoplasty from the Cornea Donor Study. Ophthalmology. 2010;117:438-444.

33. Ishii N, Yamaguchi T, Yazu H, Satake Y, Yoshida A, Shimazaki J. Factors associated with graft survival and endothelial cell density after Descemet's stripping automated endothelial keratoplasty. Sci Rep. 2016;6:25276.

34. Hayashi T, Yamaguchi T, Yuda K, Kato N, Satake Y, Shimazaki J. Topographic characteristics after Descemet's membrane endothelial keratoplasty and Descemet's stripping automated endothelial keratoplasty. PLoS One. 2017;12:e0188832.

35. Prasanth B, Dubey S, Mathur U. IOP changes after DSEK. Ophthalmology. 2010;117:1460-1461; author reply 1461-1462.

36. Moriarty AP, Spalton DJ, Shilling JS, Ffytche TJ, Bulsara M. Breakdown of the blood-aqueous barrier after argon laser panretinal photocoagulation for proliferative diabetic retinopathy. Ophthalmology. 1996;103:833-838.

37. Moriarty AP, McHugh JD, Spalton DJ, Ffytche TJ, Shah SM, Marshall J. Comparison of the anterior chamber inflammatory response to diode and argon laser trabeculoplasty using a laser flare meter. Ophthalmology. 1993;100:1263-1267.

38. Chowdhury UR, Madden BJ, Charlesworth MC, Fautsch MP. Proteome analysis of human aqueous humor. Invest Ophthalmol Vis Sci. 2010;51:49214931. 